\title{
DFT Examination of Electronic and Structural Features of Favipiravir for Iron Chelation
}

\author{
Azar Asgari Pari ${ }^{1}$ (D), Mohammad Yousefi 2,* (D) \\ 1 Department of Chemistry, Yadegar-e Imam Khomeini (RAH) Shahr-e-Rey Branch, Islamic Azad University, Tehran, Iran; \\ azarasgari2@gmail.com (A.A.P.); \\ 2 Department of Chemistry, Faculty of Pharmaceutical Chemistry, Tehran Medical Sciences, Islamic Azad University, \\ Tehran, Iran; myousefi50@ @otmail.com (M.Y.); \\ * Correspondence: myousefi50@hotmail.com (M.Y.);
}

Scopus Author ID 7004062369

Abstract: Density functional theory calculations were performed to examine electronic and structural features of favipiravir (Fav) for iron (Fe) chelation. Fav was well known for the possible medication of COVID-19; however, its mechanism of action has still been a challenging issue. Therefore, this work was done to provide information regarding the possible action of Fav for participating in the Fe chelation process. To this aim, various types of molecular and atomic descriptors were obtained to discuss the topic of this work. Obtained values of energies indicated different levels of stability for pure Fav compounds, in which such variations were also found for FavFe complexes. Molecular orbital-related features showed a different tendency to contribute to reactions for both pure and complex Fav models, in which changes of the energy levels of molecular orbitals raise the detection function of Fe for Fav compounds. Atomic-scale features also indicated direct and indirect roles of atomic sites for formations of FavFe complex models. As a consequence, the idea of Fe chelation by Fav compound was affirmed regarding the obtained results with providing detailed information for investigating the mechanism of action of Fav in treatment of COVID-19.

Keywords: favipiravir; iron chelation; molecular interaction; DFT; COVID-19.

(C) 2021 by the authors. This article is an open-access article distributed under the terms and conditions of the Creative Commons Attribution (CC BY) license (https://creativecommons.org/licenses/by/4.0/).

\section{Introduction}

Favipiravir (Fav) has become well known from the appearance of COVID-19 (coronavirus disease) in late 2019 [1-3]. Indeed, the very harmful and deathful impacts of COVID-19 on the human life system pushed almost all researchers to find a possible solution to prevent virus infection distribution and medication of infected patients [4-6]. Among such huge research activities, Fav has been introduced as one of the possible drugs for the medication of those infected patients by the coronavirus [7-9]. However, the mechanism of action and percentage of success of Fav for dealing with this mysterious disease has still been an unknown issue, and further works are required [10-12]. To this point, several works have been developed to explore various features of Fav in all media of computations or experiments [11-13]. Among these, iron $(\mathrm{Fe})$ chelation has been seen as an important mechanism of action against coronavirus infection, especially for those patients with serious problems to the health system by harmful impacts on lung tissue [14-16]. Therefore, it could be a subject of examining Fe chelation with the assistance of Fav for possible treatment of COVID-19 as described by earlier works that such challenging action could help the survival of patients [17-19]. In this regard, a detailed investigation could be performed by employing computer-based tools to examine 
electronic and structural features of the compounds at the smallest molecular and atomic scales to provide more insightful information [20-22].

Within this work, the electronic and structural features of Fav were analyzed for Fe chelation by means of performing quantum chemical calculations (Figure 1). To approach the goal, the possible existence of structures for Fav was recognized first, and their ability for Fe chelation was examined next (Figure 2). Tautomerism is a common occurrence of hydrogen atom movement among oxygen and nitrogen atoms of heterocyclic organic structures [23-25]. For Fav, such a tautomerism process could be expected to occur between two nitrogen atoms of the ring to provide suitable atomic positions for participating in Fe chelation. Consequently, evaluating molecular and atomic descriptors (Tables 1 and 2) could help sense such an idea for providing more insightful information about the treatment of mysterious COVID-19 by the assistance of Fav within Fe chelation aspect.

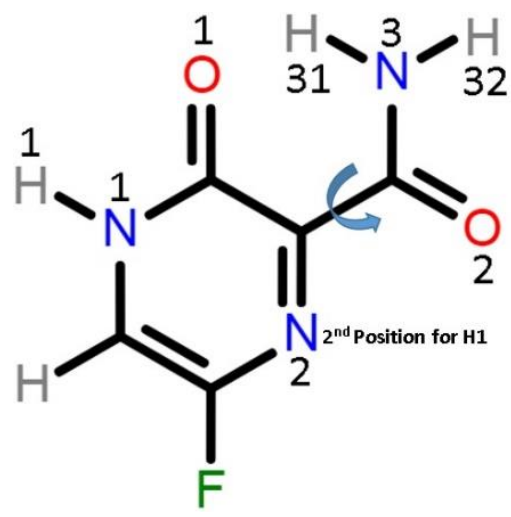

Figure 1. Favipiravir (Fav).

\section{Materials and Methods}

First, 3D models of Fav compounds were obtained by performing optimization processes to achieve stabilized structures regarding the movement of hydrogen atoms among two nitrogen atom positions of heterocyclic structures (Figures 1 and 2). By doing such processes, four models, including Fav1-Fav4, were recognized for participating in the $\mathrm{Fe}$ chelation process. Such $\mathrm{Fe}$ atomic decorated models were optimized again to characterize such features of Fe chelation by each of Fav compounds resulting in four models of Fav1Fe-Fav4Fe. Full optimization processes were performed for the models' systems employing the B3LYP/6$31 \mathrm{G}^{*}$ level of density functional of theory (DFT) as implemented in the Gaussian program [26]. As a result, the optimized models were obtained to evaluate corresponding electronic and structural features for approaching the goal of this work for examining Fav for participating in Fe chelation processes. Various types of energies, including total energy (Etot) and chelation energy (Echel) were obtained for the model systems to show the strength of obtained structural systems. Moreover, energy levels of the highest occupied molecular orbital (HOMO) and the lowest unoccupied molecular orbital (LUMO), energy gap (Egap), chemical hardness and softness $(\eta$ and $\sigma)$, and dipole moment $(\mathrm{Dm})$ were evaluated for the model systems as summarized in Table 1. HOMO and LUMO distribution patterns were also visualized for showing the frontier molecular orbitals localizations in the models' systems (Figure 2). In addition to such molecular-scale descriptors, atomic features were analyzed by calculating quadrupole coupling constants $(\mathrm{Cq})$ for the model systems' $\mathrm{H}, \mathrm{N}, \mathrm{O}$, and $\mathrm{Fe}$ atoms, as summarized in (Table 2). Earlier works indicated that such $\mathrm{Cq}$ atomic descriptors could 
recognize the electronic features of atomic sites to show variations of such properties among the investigated model systems [27-29]. To this point, such atomic descriptors were obtained here for investigating the model systems for showing the impacts of Fe chelation on the original features of Fav and how Fav could work for such function regarding the atomic scale aspects. As a consequence, the required features for examining electronic and structural features of the models' systems were provided to reach the goal of this work (Tables 1 and 2 and Figures 1 and 2).

Table 1. Molecular descriptors for the optimized models."

\begin{tabular}{|c|c|c|c|c|c|c|c|c|}
\hline Model & Etot eV & Echel eV & HOMO eV & LUMO eV & Egap eV & $\eta \mathrm{eV}$ & $\sigma \mathrm{eV}^{-1}$ & Dm Debye \\
\hline Fav1 & -16530.793 & $\mathrm{n} / \mathrm{a}$ & -6.712 & -2.658 & 4.054 & 2.027 & 0.493 & 5.906 \\
\hline Fav2 & -16530.628 & $\mathrm{n} / \mathrm{a}$ & -6.464 & -2.631 & 3.833 & 1.916 & 0.522 & 5.329 \\
\hline Fav3 & -16529.460 & $\mathrm{n} / \mathrm{a}$ & -6.104 & -2.829 & 3.276 & 1.638 & 0.611 & 8.191 \\
\hline Fav4 & -16530.418 & $\mathrm{n} / \mathrm{a}$ & -6.319 & -2.858 & 3.461 & 1.730 & 0.578 & 2.913 \\
\hline Fav1Fe & -50913.454 & -3.634 & -4.347 & -1.819 & 2.527 & 1.264 & 0.791 & 1.948 \\
\hline Fav2Fe & -50912.638 & -2.981 & -3.519 & -2.264 & 1.255 & 0.628 & 1.594 & 3.588 \\
\hline Fav3Fe & -50912.564 & -4.076 & -4.416 & -1.985 & 2.431 & 1.215 & 0.823 & 4.206 \\
\hline Fav4Fe & -50912.070 & -2.624 & -4.538 & -2.108 & 2.430 & 1.215 & 0.823 & 4.217 \\
\hline
\end{tabular}

\section{Results and Discussion}

As shown in Figure 1, the model structure of Fav could be expected for Fe chelation by the assistance of available oxygen and nitrogen sites as assigned by $\mathrm{N} 1 / \mathrm{N} 2$ and $\mathrm{O} 1 / \mathrm{O} 2$. The hydrogen atom of $\mathrm{N} 1$ could be moved to another position located at $\mathrm{N} 2$ for making possible such co-assistance of $\mathrm{N}$ and $\mathrm{O}$ atoms for Fe chelation. Moreover, the bond of the external attached group to the heterocyclic ring could be rotated to make possible such a chelation region. By such descriptions, four models of parent Fav compounds were found, as shown in Figure 2, in which other Fe chelated models were obtained for the models' systems. Available lone pairs of electrons at the atomic valence shells of $\mathrm{N}$ and $\mathrm{O}$ atoms and available vacancy of orbitals at the atomic valence shell of Fe could make possible such chelation by means of Fav compound. To this point, the models were optimized to reach the stabilized structures, and the corresponding descriptors (Tables 1 and 2) were found to analyze the investigated systems' electronic and structural features.

Based on obtained energies from optimization processes, Fav1 was the structure with the most stabilization strength among the investigated models of parent Fav compounds. The hydrogen movement and the bond rotation could change the models' features as indicated by evaluating different bond distances among Fav1 - Fav4 compounds. It is a distance variation and perturbates the electronic features of models leading by such structural features variations. In this case, the evaluated descriptors could show the effects of such models' variations in molecular and atomic scales. As mentioned for Fav1 with the highest stability among the models, the obtained molecular orbital related descriptors could affirm such achievement with the wider energy gap between HOMO and LUMO levels for the Fav1 model. As a result, each chemical hardness and softness underwent the destination of unfavorability for participating in reactions with other substances. By such achievement, the model of Fav1Fe could not be expected to be the highest stabilized model, in which energies of optimized models affirmed it. Therefore, it was necessary to examine other possibilities for Fav structure to examine other conditions of Fe chelation for the models' systems. The results indicated variation for features of the investigated models. 

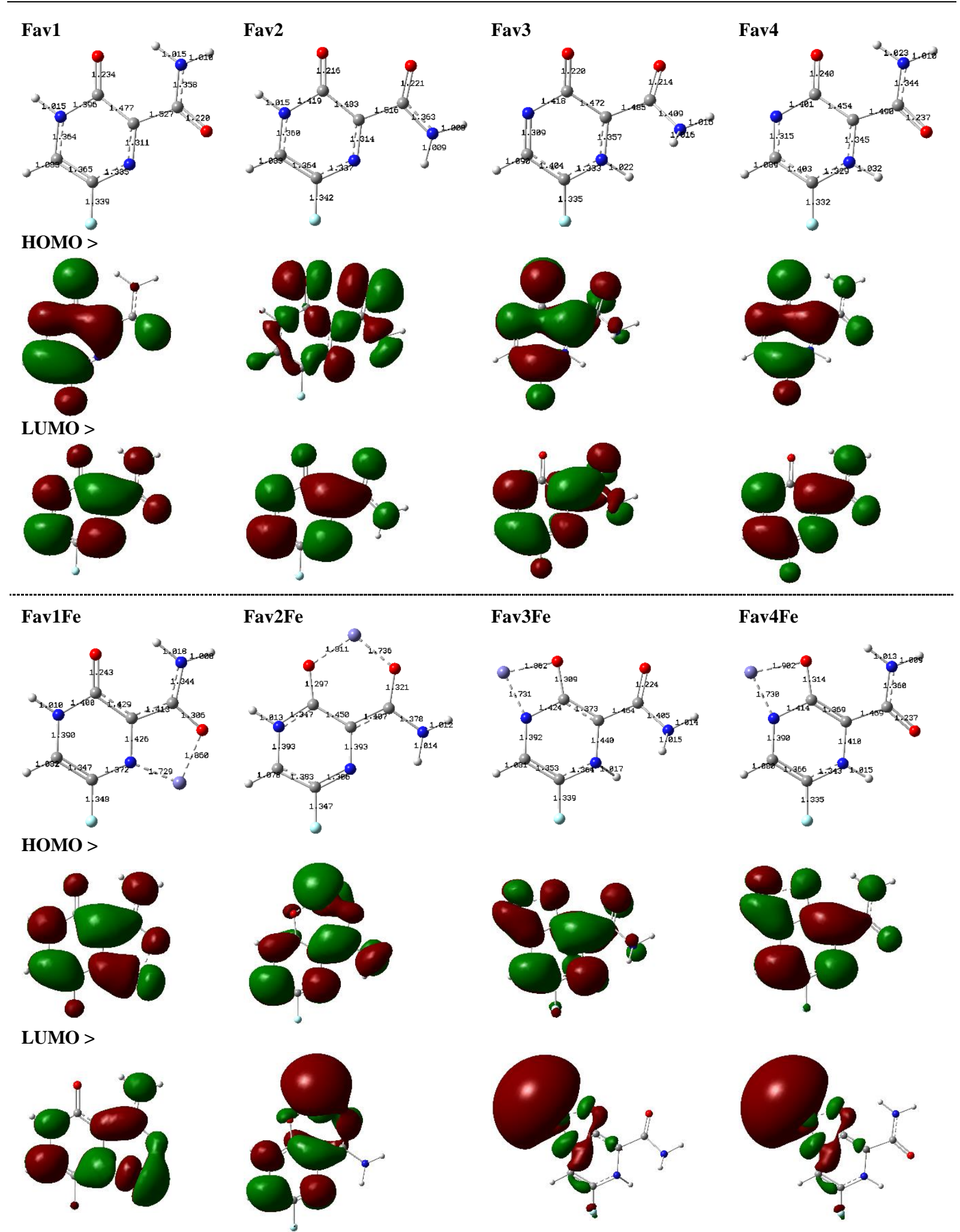

Figure 2. Optimized models of parent Fav compounds and FavFe complexes, in addition to visualized HOMO and LUMO distribution patterns. Bond distances were shown in angstrom.

Comparing the models indicated that the parent Fav3 model was found to be at the lowest stability for formation through optimization; in contrast, it was found to be at the highest stability for the Fe chelation process yielding Fav3Fe model. Such trend was affirmed by the evaluated values of molecular orbital features showing a higher possibility for contributing to reactions for the parent model. As mentioned before, even small variations in structural forms could lead to significant observation in electronic features, in which they are all identical in 
chemical formula but with many more details in a structural formula to be investigated by benefits of performing computer-based works [30-32]. By comparing energy vales for FavFe models, it could be seen that Fav3Fe was the most favorable one, whereas Fav4Fe was the most unfavorable one for the formation of Fe chelated systems. The results indicated that assistance of $\mathrm{N}$ and $\mathrm{O}$ atoms for Fe chelation could work better than two identical $\mathrm{O}$ atoms for the purpose. In addition, rotation of the external attached amide group could lead to the most favorable or the most unfavorable model of Fe chelation by Fav, as seen by what happened for Fav3Fe and Fav4Fe models. In such a case, structural and electronic features could determine a molecular model's ability to show specified functions in reaction media.

Analyzing visual representations of HOMO and LUMO distribution patterns could show a significant variation of such molecular orbital localization for HOMO of Fav2 model in comparison with other models, whereas LUMO patterns were almost identical for the model systems. Such variations were seen for localization of LUMO at the chelated Fe atom in all FavFe models, more typically for Fav3Fe and Fav4Fe models. Significant variations of HOMO and LUMO of FavFe models' energy levels compared to the parent Fav models could reveal the advantage of such a molecular model for detecting the existence of Fe in the media. In other words, the existence of Fe could be detected by means of Fav models as their electronic features of HOMO and LUMO could make them appropriate for a biosensor material. In such a case, innovating detection kits could be considered by means of small-molecule systems, avoiding the complexity of employing large-scale molecular systems. Combinations of visual representations of HOMO and LUMO and their corresponding quantities, the ability of Fav for Fe chelation were shown more or less significant with the variations of structural features. It could be remembered here that the favorability of FavFe formation was different comparing the values of obtained energies, in which such variations were found for the corresponding molecular orbital features.

Table 2. Atomic quadrupole coupling constant (Cq $\mathrm{MHz})$ descriptors for the optimized models.

\begin{tabular}{|c|c|c|c|c|c|c|c|c|c|}
\hline Model & H1 & H31 & H32 & N1 & $\mathrm{N} 2$ & N3 & 01 & $\mathbf{O 2}$ & $\mathrm{Fe}$ \\
\hline Fav1 & 0.246 & 0.231 & -0.264 & 2.669 & 5.271 & 3.844 & 8.175 & 9.208 & $\mathrm{n} / \mathrm{a}$ \\
\hline Fav2 & 0.247 & 0.259 & -0.266 & 2.715 & 4.868 & 4.316 & 9.154 & 9.831 & $\mathrm{n} / \mathrm{a}$ \\
\hline Fav3 & 0.226 & 0.252 & 0.251 & 4.849 & 1.140 & 4.152 & 9.495 & 10.096 & $\mathrm{n} / \mathrm{a}$ \\
\hline Fav4 & 0.207 & 0.214 & 0.263 & 4.880 & 0.835 & 3.565 & 8.406 & 7.766 & $\mathrm{n} / \mathrm{a}$ \\
\hline Fav1Fe & 0.259 & 0.230 & 0.267 & 4.267 & 1.926 & 4.133 & 7.745 & 7.557 & 33.307 \\
\hline Fav2Fe & 0.250 & 0.247 & 0.261 & 2.769 & 3.244 & 4.363 & 7.614 & 6.870 & 43.787 \\
\hline Fav3Fe & 0.242 & 0.245 & 0.267 & 1.884 & 3.926 & 4.380 & 7.326 & 8.207 & 32.408 \\
\hline Fav4Fe & 0.244 & 0.254 & 0.256 & 1.981 & 4.321 & 4.226 & 7.165 & 9.328 & 39.286 \\
\hline
\end{tabular}

Atomic-scale quadrupole coupling constant $(\mathrm{Cq})$ descriptors for those atoms involved in structural variation and chelation are listed in Table 2. Magnitudes of $\mathrm{Cq}$ descriptors could very well show the impact of structural variations at the atomic sites to analyze models at the lowest atomic scales [33-35]. A quick look at the obtained results of Table 2 could indicate that the atomic features of models detected effects of variations more or less significant for the models compared to each other. Hydrogen atoms did not directly contribute to Fe chelation processes; however, movements of positions for $\mathrm{H} 1$ and rotation of bond for $\mathrm{H} 31$ and $\mathrm{H} 32$ yielded variations of magnitudes in both pure Fav compounds and FavFe complexes. N1 and $\mathrm{N} 2$ atoms had contributions to the Fe chelation process, in which magnitudes of their $\mathrm{Cq}$ parameters indicated such significant contributions to the Fe chelation process. Indeed, such atomic descriptors could affirm the formation of complex models of a significant contribution of an atom to the interacting environment in detail. Such information could help for managing 
further modification of molecular structures with the requirements of specified applications. As a consequence of the results of this work, atomic-scale $\mathrm{Cq}$ descriptors showed significant roles of those atoms directly contributed to the Fe chelation model or those atoms detecting indirect effects of the occurrence of such processes the obtained results.

\section{Conclusions}

This work was performed to analyze the electronic and structural features of Fav for Fe chelation through evaluated molecular and atomic descriptors of DFT calculations. The results indicated that structural variations of parent Fav compound by the movement of $\mathrm{H}$ atom between two $\mathrm{N}$ atoms of the heterocyclic ring could provide appropriate positions for the occurrence of $\mathrm{Fe}$ chelation in addition to the rotation of bond of attached amide group to that ring structure. As a consequence, models of pure Fav compounds and dour models of FavFe complex models were obtained through performing optimization processes. The evaluated molecular and atomic scale descriptors indicated different features for the investigated models, in which Fav1 was at the highest stability and Fav3 was at the lowest stability, whereas Fav3Fe was at the highest favorability of formation for the investigated models. Moreover, variations of molecular orbital-related features yielded different tendencies of contributing to reaction for pure Fav compounds, in which such variations could also help for detection purposes of Fe by means of small molecules avoiding the complexity of large molecular systems. Additional achievements by atomic-scale descriptors indicated the roles of atoms with direct and indirect contributions to Fe chelation processes, in which the systems were analyzed regarding the importance of atomic roles. As a final remark, Fe chelation could occur with the assistance of Fav compound with details of the formation of FavFe complexes to further investigate its mechanism of action in treating COVID-19.

\section{Funding}

This research received no external funding.

\section{Acknowledgments}

This research received no external acknowledgments.

\section{Conflicts of Interest}

The authors declare no conflict of interest.

\section{References}

1. Naydenova, K.; Muir, K.W.; Wu, L-F.; Zhang, Z.; Coscia, F.; Peet, M.J.; Castro-Hartmann, P.; Qian, P.; Sader, K.; Dent, K.; Kimanius, D. \& al. Structure of the SARS-CoV-2 RNA-dependent RNA polymerase in the presence of favipiravir-RTP. Proceedings of the National Academy of Sciences 2021, 118, e2021946118, https://doi.org/10.1073/pnas.2021946118.

2. Takahashi, H.; Iwasaki, Y.; Watanabe, T.; Ichinose, N.; Okada, Y.; Oiwa, A.; Kobayashi, T.; Moriya, M.; Oda, T. Case studies of SARS-CoV-2 treated with favipiravir among patients in critical or severe condition. International Journal of Infectious Diseases 2020, 100, 283-285, https://doi.org/10.1016/j.ijid.2020.08.047.

3. Harismah, K.; Mirzaei, M. Favipiravir: structural analysis and activity against COVID-19. Advanced Journal of Chemistry B 2020, 2, 55-60, https://doi.org/10.33945/SAMI/AJCB.2020.2.3.

4. Ozkendir, O.M.; Askar, M.; Kocer, N.E. Influence of the epidemic COVID-19: an outlook on health, business and scientific studies. Lab-in-Silico 2020, 1, 26-30, https://doi.org/10.22034/labinsilico20011026. 
5. Harismah, K.; Mirzaei, M. COVID-19: a serious warning for emergency health innovation. Advanced Journal of Science and Engineering 2020, 1, 32-33, https://doi.org/10.22034/AJSE.2012032.

6. Arshizadeh, S.; Gorgani, S.H.; Taheri, P.; Givgol, M.; Shahrokhi, S.; Abdalisousan, A. The impact of COVID19 on oil supply in the short term. Advanced Journal of Science and Engineering 2021, 2, 120-135, https://doi.org/10.22034/advjscieng21022120.

7. Joshi, S.; Parkar, J.; Ansari, A.; Vora, A.; Talwar, D.; Tiwaskar, M.; Patil, S.; Barkate, H. Role of favipiravir in the treatment of COVID-19. International Journal of Infectious Diseases 2021, 102, 501-508, https://doi.org/10.1016/j.ijid.2020.10.069.

8. Dabbous, H.M.; Abd-Elsalam, S.; El-Sayed, M.H.; Sherief, A.F.; Ebeid, F.F.; Abd El Ghafar, M.S.; Soliman, S.; Elbahnasawy, M.; Badawi, R.; Tageldin, M.A. Efficacy of favipiravir in COVID-19 treatment: a multicenter randomized study. Archives of Virology 2021, 166, 949-954, https://doi.org/10.1007/s00705-02104956-9.

9. Lou, Y.; Liu, L.; Yao, H.; Hu, X.; Su, J.; Xu, K.; Luo, R.; Yang, X.; He, L.; Lu, X.; Zhao, Q. \& al. Clinical outcomes and plasma concentrations of baloxavir marboxil and favipiravir in COVID-19 patients: an exploratory randomized, controlled trial. European Journal of Pharmaceutical Sciences 2021, 157, 105631, https://doi.org/10.1016/j.ejps.2020.105631.

10. Hashemian, S.M.; Farhadi, T.; Velayati, A.A. A review on favipiravir: the properties, function, and usefulness to treat COVID-19. Expert Review of Anti-Infective Therapy 2021, 19, 1029-1037, https://doi.org/10.1080/14787210.2021.1866545.

11. Boretti, A. Favipiravir use for SARS CoV-2 infection. Pharmacological Reports 2020, 72, 1542-1552, https://doi.org/10.1007/s43440-020-00175-2.

12. Shrestha, D.B.; Budhathoki, P.; Khadka, S.; Shah, P.B.; Pokharel, N.; Rashmi, P. Favipiravir versus other antiviral or standard of care for COVID-19 treatment: a rapid systematic review and meta-analysis. Virology Journal 2020, 17, 141, https://doi.org/10.1186/s12985-020-01412-z.

13. Ashjaee, Y.; Zandi, H. Molecular analysis of 5-COR derivatives of uracil and evaluating their affinity against the MPro target of COVID-19. Advanced Journal of Science and Engineering 2021, 2, 79-85, https://doi.org/10.22034/advjscieng21022079.

14. Vlahakos, V.D.; Marathias, K.P.; Arkadopoulos, N.; Vlahakos, D.V. Hyperferritinemia in patients with COVID-19: an opportunity for iron chelation? Artificial Organs 2021, 45, 163-167, https://doi.org/10.1111/aor.13812.

15. Carota, G.; Ronsisvalle, S.; Panarello, F.; Tibullo, D.; Nicolosi, A.; Li Volti, G. Role of iron chelation and protease inhibition of natural products on COVID-19 infection. Journal of Clinical Medicine 2021, 10, 2306, https://doi.org/10.3390/jcm10112306.

16. Abobaker, A. Reply: iron chelation may harm patients with COVID-19. European Journal of Clinical Pharmacology 2021, 77, 267-268, https://doi.org/10.1007/s00228-020-02988-9.

17. Ghasemiyeh, P.; Mohammadi-Samani, S. Iron chelating agents: promising supportive therapies in severe cases of COVID-19? Trends in Pharmaceutical Sciences 2020, 6, 65-66, https://doi.org/10.30476/tips.2020.86274.1047.

18. Qiu, J.; Ma, J.; Zhang, S.; Han, J.; Liu, S. Promoting platelets is a therapeutic option to combat severe viral infection of the lung. Blood Advances 2020, 4, 1640-1642, https://doi.org/10.1182/bloodadvances.2020001669.

19. Bou-Fakhredin, R.; Daadaa, H.; Koussa, S.; Abou Nasr, T.; Noun, P.; Taher, A.T. SARS-CoV-2 infection in patients with $\beta$-thalassemia: experience from Lebanon. American Journal of Hematology 2021, 96, E285E288, https://doi.org/10.1002/ajh.26211.

20. Ghamsari, P.A.; Samadizadeh, M.; Mirzaei, M. Halogenated derivatives of cytidine: Structural analysis and binding affinity. Journal of Theoretical and Computational Chemistry 2020, 19, 2050033, https://doi.org/10.1142/S0219633620500339.

21. Mirzaei, M.; Karimi, E.; Yousefi, M. BN nanoflake for hazardous $\mathrm{SO}_{2}$ gas capturing: DFT study. Biointerface Research in Applied Chemistry 2022, 12, 359-365, https://doi.org/10.33263/BRIAC121.359365.

22. Mirzaei, M.; Harismah, K.; Da'i, M.; Salarrezaei, E.; Roshandel, Z. Screening efficacy of available HIV protease inhibitors on COVID-19 protease. Journal of Military Medicine 2020, 22, 100-107, https://doi.org/10.30491/JMM.22.2.100.

23. Gilani, A.G.; Taghvaei, V.; Rufchahi, E.M.; Mirzaei, M. Tautomerism, solvatochromism, preferential solvation, and density functional study of some heteroarylazo dyes. Journal of Molecular Liquids 2019, 273, 392-407, https://doi.org/10.1016/j.molliq.2018.10.054. 
24. Yaraghi, A.; Ozkendir, O.M.; Mirzaei, M. DFT studies of 5-fluorouracil tautomers on a silicon graphene nanosheet. Superlattices and Microstructures 2015, 85, 784-788, https://doi.org/10.1016/j.spmi.2015.05.053.

25. Kouchaki, A.; Gülseren, O.; Hadipour, N.; Mirzaei, M. Relaxations of fluorouracil tautomers by decorations of fullerene-like SiCs: DFT studies. Physics Letters A 2016, 380, 2160-2166, https://doi.org/10.1016/j.physleta.2016.04.037.

26. Frisch, M.J.; Trucks, G.W.; Schlegel, H.B.; Scuseria, G.E.; Robb, M.A.; Cheeseman, J.R.; Scalmani, G.; Barone, V. \& al. Gaussian 09. Gaussian, Inc., Wallingford, CT 2009.

27. Mirzaei, M.; Meskinfam, M.; Yousefi, M. A cytosine-assisted carbon nanotubes junction: DFT studies. Superlattices and Microstructures 2012, 52, 158-164, https://doi.org/10.1016/j.spmi.2012.04.018.

28. Mirzaei, M.; Meskinfam, M.; Yousefi, M. Covalent hybridizations of carbon nanotubes through peptide linkages: a density functional approach. Computational and Theoretical Chemistry 2012, 981, 47-51, https://doi.org/10.1016/j.comptc.2011.11.043.

29. Aramideh, M.; Mirzaei, M.; Khodarahmi, G.; Gülseren, O. DFT studies of graphene-functionalised derivatives of capecitabine. Zeitschrift für Naturforschung A 2017, 72, 1131-1138, https://doi.org/10.1515/zna-2017-0290.

30. Zandi, H.; Harismah, K. Computer-based tools for structural characterizations and activity specifications of natural products: a quick review. Lab-in-Silico 2021, 2, 50-54, https://doi.org/10.22034/labinsilico21021050.

31. Fallahpour, F.; Ariaei, S. Computational investigation of B6 particle for $\mathrm{H}_{2} \mathrm{~S}$ capturing. Advanced Journal of Science and Engineering 2021, 2, 31-35, https://doi.org/10.22034/advjscieng21021031.

32. Ariaei, S. DFT calculations of a cubic B4N4 cubane-like particle for CO gas adsorption. Advanced Journal of Science and Engineering, 2021, 2, 93-98, https://doi.org/10.22034/advjscieng21022093.

33. Mirzaei, M.; Harismah, K.; Jafari, E.; Gülseren, O.; Rad, A.S. Functionalization of (n, 0) CNTs (n= 3-16) by uracil: DFT studies. European Physical Journal B 2018, 91, 14, https://doi.org/10.1140/epjb/e2017-80404-1.

34. Mirzaei, M.; Gülseren, O.; Hadipour, N. DFT explorations of quadrupole coupling constants for planar 5fluorouracil pairs. Computational and Theoretical Chemistry 2016, 1090, 67-73, https://doi.org/10.1016/j.comptc.2016.06.004.

35. Harismah, K.; Dhumad, A.M.; Ibraheem, H.S.; Zandi, H.; Majeed, H.J. A DFT approach on tioguanine: exploring tio-tiol tautomers, frontier molecular orbitals, IR and UV spectra, and quadrupole coupling constants. Journal of Molecular Liquids 2021, 334, 116018, https://doi.org/10.1016/j.molliq.2021.116018. 